\title{
Design and Evaluation of Two Robotic Furnishings Partnering with Each Other and Their Users to Enable Independent Living
}

\author{
Siddharth Verma \\ Clemson University \\ Clemson, South Carolina \\ siddhav@g.clemson.edu \\ Dixit Nahar \\ Clemson University \\ Clemson, South Carolina \\ dnahar@g.clemson.edu \\ Yixiao Wang
Cornell University
Ithaca, New York
yw697@cornell.edu
}

\author{
Phanideep Gonthina \\ Clemson University \\ Clemson, South Carolina \\ pgonthi@g.clemson.edu \\ Johnell O. Brooks \\ Clemson University \\ Clemson, South Carolina \\ jobrook@g.clemson.edu \\ Carlos de Aguiar \\ Cornell University \\ Ithaca, New York \\ ca449@cornell.edu
}

\author{
Zachary Hawks \\ Clemson University \\ Clemson, South Carolina \\ zhawks@g.clemson.edu \\ Ian D. Walker \\ Clemson University \\ Clemson, South Carolina \\ iwalker@g.clemson.edu \\ Keith E. Green \\ Cornell University \\ Ithaca, New York \\ keg95@cornell.edu
}

\begin{abstract}
We introduce a pair of domestic, robotic furnishings aimed at improving the ability of people to live and work independently. The robotic pair-a mobile, robot-cube and a continuum-robotic lampwork together with their human cohabitants to perform routine tasks of daily living enumerated in the 'CS-PFP10' protocol used by rehabilitation therapists to evaluate the capacity for independent living. The iterative design and basic behaviors of the robot pair are considered in this paper, as are results from a formative user evaluation involving older adults and a second study involving twelve clinical staff from a rehabilitation hospital. Finally, we offer recommendations that generalize to related efforts. As robots will inevitably become part of domestic routines, reporting on this robot pair serves as a design exemplar for future development of domestic robots that enable and dignify older individuals.
\end{abstract}

\section{CCS CONCEPTS}

- Human-centered computing $\rightarrow$ Interaction design process and methods; • Social and professional topics $\rightarrow$ Seniors; • Computer systems organization $\rightarrow$ Robotics;

\section{KEYWORDS}

Enabling Technology, Home-Based Lifestyle Support, Robotic Furniture, Design, Rehabilitation, Usability, Acceptability

\section{ACM Reference Format:}

Siddharth Verma, Phanideep Gonthina, Zachary Hawks, Dixit Nahar, Johnell O. Brooks, Ian D. Walker, Yixiao Wang, Carlos de Aguiar, and Keith E. Green.

Permission to make digital or hard copies of all or part of this work for personal or classroom use is granted without fee provided that copies are not made or distributed for profit or commercial advantage and that copies bear this notice and the full citation on the first page. Copyrights for components of this work owned by others than ACM must be honored. Abstracting with credit is permitted. To copy otherwise, or republish, to post on servers or to redistribute to lists, requires prior specific permission and/or a fee. Request permissions from permissions@acm.org.

PervasiveHealth '18, May 21-24, 2018, New York, NY, USA

(C) 2018 Association for Computing Machinery.

ACM ISBN 978-1-4503-6450-8/18/05 . .\$15.00

https://doi.org/10.1145/3240925.3240978
2018. Design and Evaluation of Two Robotic Furnishings Partnering with Each Other and Their Users to Enable Independent Living. In PervasiveHealth '18: 12th EAI International Conference on Pervasive Computing Technologies for Healthcare (PervasiveHealth '18), May 21-24, 2018, New York, NY, USA. ACM, New York, NY, USA, 10 pages. https://doi.org/10.1145/3240925. 3240978

\section{INTRODUCTION}

The opportunity for domestic robots is compounding [16] as older adults account for a larger segment of the overall human population, as a decreasing segment of the human population is able to care for and pay for the well-being of older and clinical populations, and as people overwhelmingly want to stay in their own homes as they age [5]. In the case of the United States, it is estimated that, by 2025 , there will be a shortage of physicians and nurses, and by 2040, there will be more than 79.7 million adults who will be age 65 or older-a 92 percent increase from 2011 [2]. Domestic robots have the potential to reduce the burden on both care providers and tax payers, while meeting the practical needs and ensuring the well-being of an increasingly aging population.

Predominately, the materialization of domestic robots has taken two forms: the relatively low-cost, single-function robot (most visibly, the vacuum cleaning Roomba), and the out-of-reach and not yet primed humanoid servant (among them, the hulking humanoid robot, PR2). If single-function domestic robots come to proliferate, however, our homes will be littered with them-an untenable living condition. As for the possibility of an in-home, "humanoid" service robot, there are doubts about when or if an affordable, reliable humanoid assistant of vast capabilities and intelligence is coming to anyone's home soon [16],[22]. Arguably, humanoid robots have had difficulty accomplishing things we do instinctively or with little effort, like picking up after themselves or picking themselves up after a fall-what we know as Moravec's paradox [22]. Furthermore, data (as reported in e.g. [13],[35] have suggested that while people may welcome a service robot to compensate for their reduced capacities, they don't care all that much whether the robot looks or acts particularly human or animal-like. 


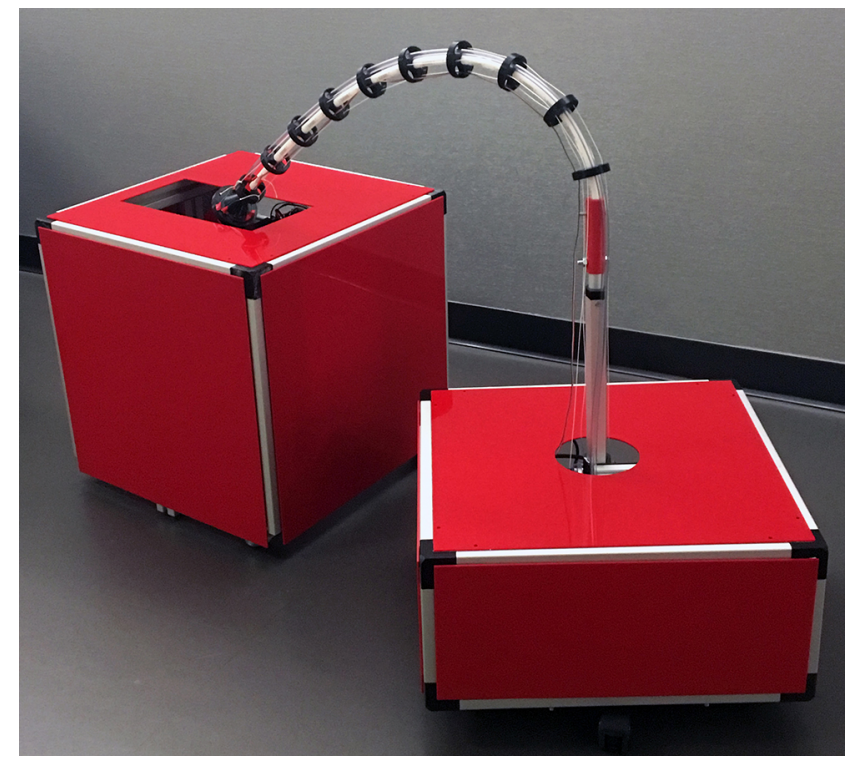

Figure 1: h+cube (left) and h+lamp (right) - prototype-2.

Despite a surprising receptiveness to new technologies enabling independent living in the home [6],[8],[17],[25], robotics research for health and eldercare applications has tended to focus either on rehabilitation robotics (e.g. [20], robot-assisted surgery (e.g. [1], prosthetics (e.g. [10]), as replacements for humans engaged in healthcare-related activity (e.g. [29]), for companionship [34], or to perform as a single-body mobile robot a few, simple domestic tasks [12],[14]. At the same time, the domestic space itself-even dwellings built on a one-size-fits-all, 'universal design' approach [26]-remain essentially conventional, low-tech, and maladaptive to the dramatic changes in human demographics and to the lifecycles of their inhabitants.

In a multi-year, multi-project ambition we call home+, the design challenge of our expanded design-research team (which includes human factors psychologists and domain-specific, healthcare providers) is to understand populations that might benefit the most (elders and care providers) and design for them, and with them, not a single robot helper but a suite of domestic robotic furnishings that is multi-functional, interoperable, low-cost, and enabling independent living at home. Our ambition is informed by wide-ranging input, including furniture design through history [15], and guidelines for designing domestic robots [3]

At its core, home+ is a collection of networked, cyber-physical, domestic furnishings, each exhibiting different functionalities tuned for an array of purposes to support routine domestic tasks and, generally, to exploit an opportunity to improve the lives of their human cohabitants. While there have been efforts to develop robotic furnishings (e.g. [11],[19],[30],[38] outside our research lab, closest to our vision of robotic furniture supporting domestic routines is the concept of the "Intelligent Sweet Home" [28] from KAIST (the Korea Advanced Institute of Science and Technology); however, in this research effort, the only robotic component receiving significant attention, to our knowledge, is a hoist for transferring users to and from their beds-a robotic device serving a singular, practical function [28].

\section{SYSTEM DESCRIPTION AND CHARACTERIZATION}

This paper presents, for the first time, our design and characterization of a pair of robots we call $h+c u b e$ and $h+l a m p$ (Figure 1), the latest iteration of the larger home+ project

\subsection{Overview of $h+c u b e, h+l a m p$, and home+}

The core components of home $+(\mathrm{h}+$ cube and $\mathrm{h}+\mathrm{lamp}$, included $)$ are of the form and basic functionality of commonplace furniture found in an ordinary house, such as a side-table and floor lamp. But unlike commonplace furnishings, home+ furnishings are characterized (in the words of William Mitchell) as "geographically distributed assemblages of diverse, highly, specialized, intercommunicating artifacts" that render the physical environment "a robot for living in" [24]. Similarly, we take inspiration for home+ from former Wired editor Kevin Kelly in his vision of a future artificial "ecology" of intelligent "rooms stuffed with co-evolutionary furniture" and a "mob of tiny smart objects," all having an "awareness of each other, of themselves, and of me" [18]. Composed of multiple domestic artifacts of familiar form and basic function, the distributed environment of home+ manifests something of the behavior of swarms and also subscribes in-concept to the notion of the artificial life community that, "in living systems, the whole is more than the sum of its parts” [21].

Indeed, we conceptualize the "animated" furniture of home+ and their human users as "cohabitants" sharing a home together. home+ strives to empower people to remain in their homes for as long as possible, even as their physical capabilities alter over time, and, in more grave circumstances, to afford people some semblance of feeling at home as users move between their dwellings outfitted with home+ and assisted-care facilities equipped in the same way.

\subsection{Previous work}

$\mathrm{h}+$ cube and $\mathrm{h}+$ lamp are informed by two home+ efforts previously reported: home+ envisioned as a patient room of interoperable robotic furnishings [31], and our Assistive Robotic Table [32] that features a continuum robotic surface for rehabilitating the upper limbs of post-stroke patients [23]. More recently, we reported on a rapidly prototyped, interoperable robotic chair, wall, and table that permitted us further exploration of home+ [9]. Building on these earlier efforts, $h+$ cube and $h+l a m p$ expand the home+ ambition to two robotic furnishings-a cubic side-table and a floor lamp-that work cooperatively with each other and their human cohabitants to accomplish routine, domestic tasks that define, for healthcare providers, the capacity for independent living at home. $h+$ cube and $\mathrm{h}$ +lamp are designed to work as complements within the volume of the typical room of the home or workplace: $h+$ cube engages in tasks within a spatial volume bounded by a room's floor, extending upwards to approximately hip or table height; while h+lamp engages in tasks from hip or table height, extending upwards to nearly the ceiling of a typical room. Both robots are designed to enable an individual in a chair, on a bed, or in a wheelchair-at home and at work-to perform routine tasks defined by the "Continuum Scale 

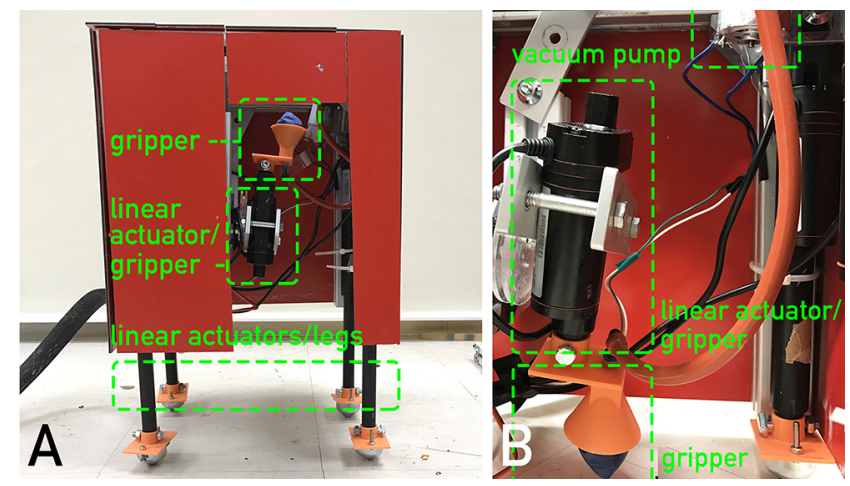

Figure 2: h+cube (prototype-1): (A) exterior-panel removed; (B) interior detail.

Physical Functional Performance” ('CS-PFP10') [7], a widely-used metric for independent living.

Before introducing this metric in the experiments section that follows, we describe the design and characterization of the two robots, and their key operational concepts.

\section{$2.3 \mathrm{~h}+$ cube design}

The fundamental purpose of $h+$ cube (Figure 2) is to perform tasks associated with the floor of the home or workplace, whether it be picking-up objects from the floor and transporting these objects either to storage or to the user, sweeping and vacuuming the floor, or moving laundry and groceries across the floor. h+lamp is the active mobile robot of this robot pair; its partner $h+$ cube moves about only by way of a human cohabitant physically moving it, or by it being coupled to and tugged by h+lamp.

The red-colored body of $h+$ cube measures $46.65 \mathrm{~cm}$ (18.37 in) along any length, and is encased in composite panels having a polyethylene core with faces of aluminum. Each of $h+$ cube's four legs is a linear actuator having a stroke length of $15.24 \mathrm{~cm}$ (6 in). Inside the cube (Figure 2.B) is a linear actuator with a gripper of our construction, based on the jamming of granular materials [37]. This actuator-gripper assembly rotates 180 degrees by way of a servo motor. The small $12 \mathrm{~V}$ vacuum pump offers a surprisingly powerful 32PSI suction. We fabricated multiple components of the $h+$ cube prototype using a 3D printer and a $\mathrm{CNC}$ router working from our digital shop drawings.

\section{$2.4 h$ +lamp design and characterization}

In our prior design efforts for home+, we recognized opportunities for advancing continuum robotics [36], machines with smooth, compliant backbones that render their movement fluid, natural, and more adaptive and life-like. The smooth movement and compliance of continuum robots lend them to intimate and elicited interactions with human users [36]. Our ART therapy surface [15] is representative of continuum robotics, capable of contributing to the formation of adaptive and inherently safe physical spaces within the built environment. While ART relied on pneumatic actuators, $h+l a m p$ is a continuum-robot trunk using motor-driven tendons to better match

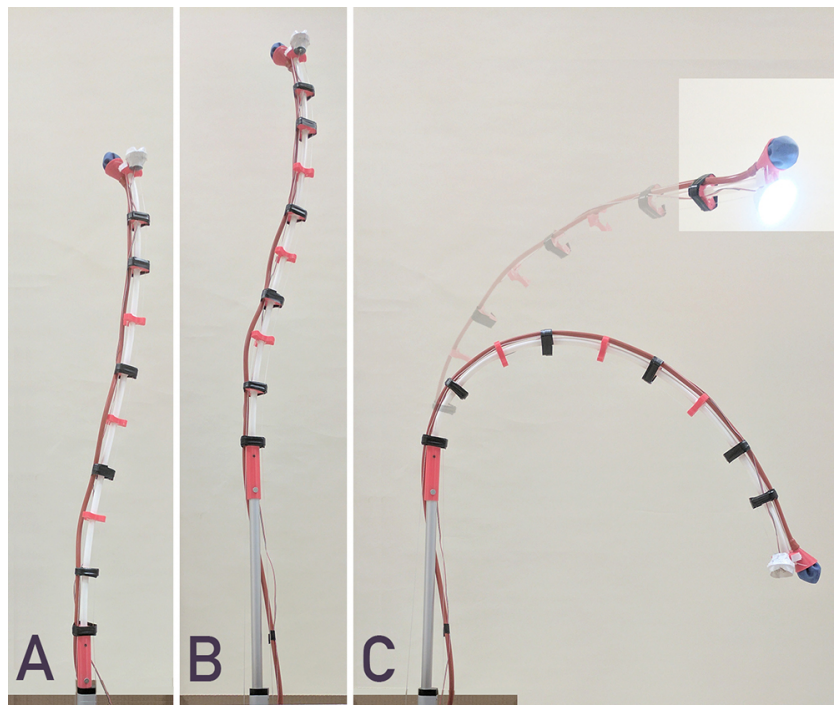

Figure 3: h+lamp (prototype-1): (A) fully rectracted; (B) fully extended; (C) fully bent and shown with lamp on.

the power sources available in typical homes, and to be more quiet and non-intrusive for human cohabitants.

The control of continuum robots-the use of sensors, actuators, and algorithms to configure them-is a non-trivial matter undertaken by the authors previously (e.g. for the ART surface [23]). Unlike the control of finite joints in rigid-link robots, the control of continuum robots involves controlling not only bending but also the extension and contraction of the entire physical mass. h+lamp's tendon-actuated trunk represents a novel form of robot in humancentered applications, as well as an innovative category of robotics for healthcare and wellbeing-not rehabilitation, not robot-assisted surgery, not prosthetics, but the enabling of independent living and working.

Specifically, h+lamp (Figures 3 and 4) is intended to perform tasks associated with the upper-half of a room's volume, whether it be removing or placing objects from/on upper shelves or cabinets, transporting objects across kitchen counters, desks, and other work surfaces, or helping users remove and put on a coat. In design, $\mathrm{h}+$ lamp has a flexible backbone of $25 \mathrm{~mm}$ diameter PEX piping. Mounted along this backbone are 3D-printed spacers (Figure 4.Bdetail) positioning three steel tendons a short distance from, and running along the PVC backbone. DC motors mounted in the lamp's base (Figure 4.A) drive each of the three tendons. This flexible assembly is mounted to a linear actuator that is firmly anchored to the lamp's mobile base. The height of the composite trunk assembly, when fully extended towards the ceiling (Fig.3.B), is $193 \mathrm{~cm}$ (76 in) from the floor; when the trunk is fully bent towards the floor (Fig.3.C), its extreme tip is $71 \mathrm{~cm}$ (28 in) from the floor. At the extreme tip of the trunk, an array of LED lights serve as task lighting, and the same gripper found on $h+$ cube is mounted. 


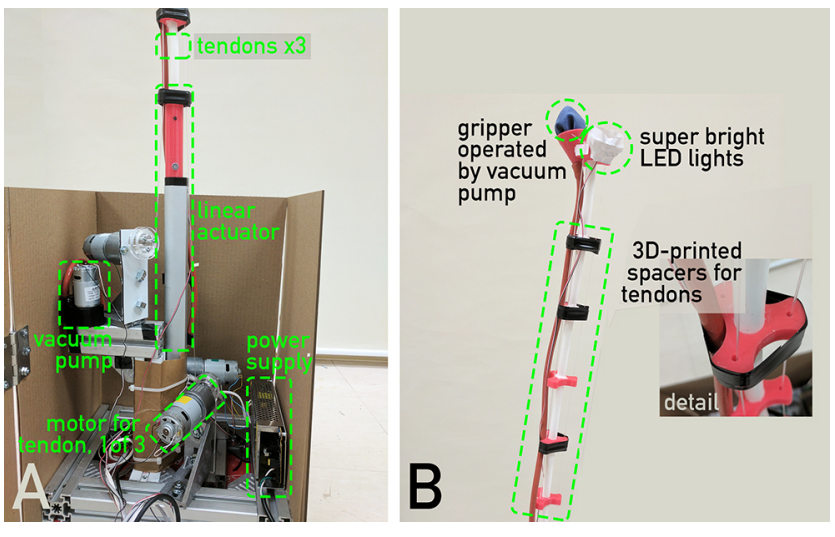

Figure 4: h+lamp (prototype-1): (A) base in detail; (B) extremity of lamp arm, with a detail showing spacers.

\subsection{Replicability}

Replicability is arguably one measure of the contribution of any design-focused paper reporting on a novel technology. By "replicability," we mean that, given the description of the design process as presented in the paper, a research team with comparable expertise can replicate the artifact elaborated in the paper without significant consultation from the authors. For the home+ pair of robots, we demonstrated just that: a full draft of this paper was used as the blueprint by half of our research team, located 1000 miles away from the partnering lab that first constructed the robot pair, to precisely replicate the two robots for concurrent and future experimentation in both labs. We offer the design process reported in this section as a design exemplar intending to help inform the research process of other design teams also engaged in developing assistive technologies.

\section{LAB EXPERIMENTS}

We conducted lab experiments with $\mathrm{h}+$ cube and $\mathrm{h}+$ lamp (prototype 1) to determine if the two robots-working separately, cooperatively, and with and without their human cohabitant(s)-could accomplish routine, domestic tasks enumerated as CS-PFP10, the Continuous Scale Physical Functional Performance-10 (Table 1) used by healthcare providers. CS-PFP10 has been proven [7] to assess balance, coordination, functional mobility, gait, general health, strength, and upper extremity function with great validity and reliability for middle-aged adults, older adults, nonagenarians, manual wheelchair users, those with coronary heart disease, fibromyalgia, Parkinson's disease, and those who suffered a stroke.

Routinely, CS-PFP10 is used to evaluate individuals to determine if they are capable of living independently in their homes; the individual is asked to perform, in a domestic setting at a healthcare facility, the ten tasks while being evaluated by medical staff trained to perform this evaluation. Co-author Johnell Brooks is an expert in using CS-PFP10 with older hospital patients and established a home lab of our team's design within the Roger C. Peace Rehabilitation Hospital of the Greenville Health System (South Carolina, USA)our clinical research partner for home+ research.
Table 1: CS-PFP10 Tasks for Independent Living

1. Carrying a weighted pot
2. Donning/removing a jacket
3. Placing and removing a sponge from a shelf
4. Transferring clothes
5. Vacuuming
6. Sweeping the floor
7. Climbing stairs
8. Picking scarves off the floor
9. Carrying groceries
10. Six-minute walk

Table 2: Overview of the four experiments

\begin{tabular}{|c|c|c|c|c|}
\hline & $\begin{array}{l}\text { TASK } \\
\text { START }\end{array}$ & $\begin{array}{l}\text { OBJECT } \\
\text { PROPERTIES }\end{array}$ & TASK END & INTERACTION \\
\hline A & $\begin{array}{l}\text { object on } \\
\text { floor }\end{array}$ & soft & $\begin{array}{l}\text { object stored } \\
\text { on shelf }\end{array}$ & $h+c u b e$ (alone) \\
\hline B & $\begin{array}{l}\text { object on } \\
\text { floor }\end{array}$ & hard, slender & $\begin{array}{l}\text { object to } \\
\text { user }\end{array}$ & $h+c u b e$-human \\
\hline $\mathrm{C}$ & $\begin{array}{l}\text { object on } \\
\text { shelf }\end{array}$ & hard, smooth & $\begin{array}{l}\text { object in } \\
\text { microwave }\end{array}$ & $h+$ lamp-human \\
\hline $\mathrm{D}$ & $\begin{array}{l}\text { object on } \\
\text { floor }\end{array}$ & hard, smooth & $\begin{array}{l}\text { object to } \\
\text { sink }\end{array}$ & $\begin{array}{l}h+c u b e- \\
h+l a m p-h u m a n\end{array}$ \\
\hline
\end{tabular}

In our experiments (see Table 2), we assessed the ability of the two robots and human users to perform CS-PFP10 tasks. Of the ten tasks of the CS-PFP10, "7. Climbing stairs" and "10. Six-minute walk" were considered outside our objective, as stair lifts, canes, and walkers are commonplace aids for accomplishing stair climbing and walking. Instead, we focused our experiments on the remaining eight tasks of the CS-PFP10, which we distilled further as four "performances" (described below) that suggest the capacity for accomplishing all eight of these tasks. For the experiments reported here, we were mindful that our user might be fully dependent upon a manual wheelchair-a realistic and challenging target-user population for our system. The research team used two wireless control boxes of our own design to control the robots, recognizing that these experiments are focused on functionality of the robot pair, and not (yet) their control. In future work, we intend to explore three control strategies for home+: user control, autonomous robot control, and a mix of user and autonomous control. We will explore these controls together with healthcare professions, as the larger home+ team designs, implements, and evaluates home+.

\subsection{Picking up an object and delivering it to storage}

"Challenge-A" for the two robots was to accomplish "8. Picking scarves off the floor." We elected to work with socks, which are as soft as scarves but more plentiful in most homes. To accomplish Challenge- $\mathrm{A}, \mathrm{h}+$ cube elevated itself using its four linear actuators (Figure 5.A), hovering over the sock (Figure 5.B), and lowered itself so that its gripper could grab the sock (Figure 5.C). The sock was then rotated 90-degrees (Figure 5.D), and inserted into a shelf 

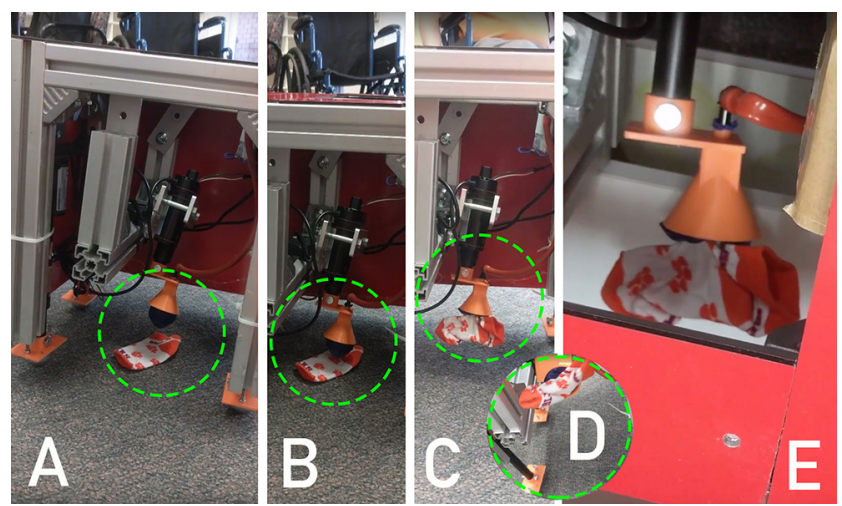

Figure 5: $h+$ cube picks-up a sock and delivers it to a shelf inside a walk-in closet.
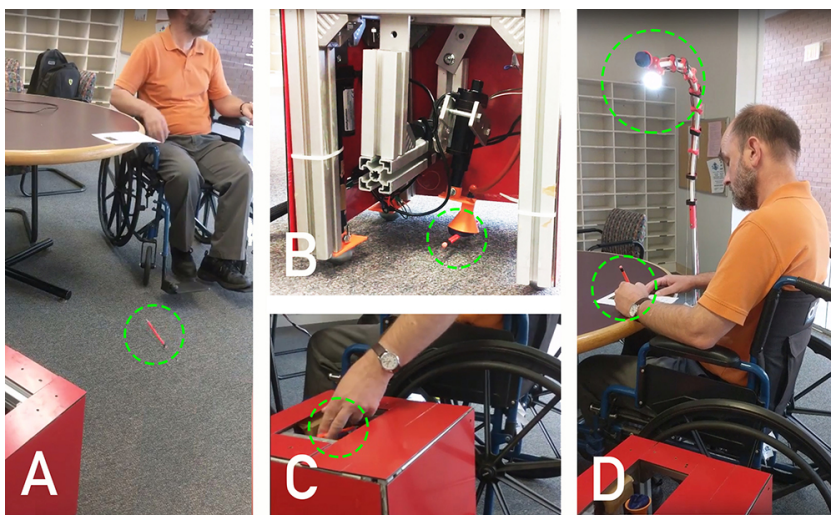

Figure 6: $h+c u b e$ collects a pencil and delivers it to the user. (h+lamp then provides task lighting for the user.)

(Figure 5.E) by the linear actuator that extends the gripper's reach. Challenge-A demonstrates how one of the two robots can collect soft objects from the floor and place such objects in convenient storage locations within the domestic environment.

We designed a simple basket that sits on top of $h+$ cube, sized to accommodate folded shirts (laundry) or a bag of groceries. $h+$ cube thus serves as an unobtrusive vehicle for transporting laundry and groceries across the threshold of the house and from room to room. As $\mathrm{h}+$ cube is able to elevate itself, the basket of laundry items or groceries it transports can more easily be handled by the human user, seated in a wheelchair. We could envision the basket dimensions of $\mathrm{h}+$ cube being standardized for laundry and grocery delivery services, given the needs of an expanding, older demographic. In any case, it is not difficult to see that $\mathrm{h}+$ cube can accomplish two more CS-PFP10 tasks: "4. Transferring clothes" and "9. Carrying groceries."

\subsection{Picking up an object and handing it to a user}

"Challenge-B" for the two robots was to accomplish a variation of "8. Picking scarves off the floor," only this time the object was a slender, hard object (a pencil) that the user dropped. We wanted to learn how the system operated with a very different form factor and rigidity than a sock. To do this, $\mathrm{h}+$ cube performed the same initial behaviors as for the sock (Figures 6.A and 6.B); but once the gripper collected the pencil, the pencil was flipped 180 degrees (from facing the floor to facing the ceiling), and elevated by linear actuator so that the user could capably grab it (Figure 6.C), and resume work under the task light offered by $h+l a m p$ (Figure 6.D). Challenge-B demonstrates how one of the two robots can collect from the floor a very different object than the sock of the previous challenge, and deliver it the human cohabitant.

We designed, but have yet to implement in $\mathrm{h}+$ cube, a system in which the user could swap-out the gripper for other tools such as a vacuum and a brush, to perform two more CS-PFP10 tasks: "5. Vacuuming" and "6. Sweeping the floor." Selecting an alternate tool would be convenient for our user, given that the tools, mounted on a rotating plate, could be presented to the user in the same way described here for the pencil, so that the user can simply rotate the collected tools to the one matched to the task.

\subsection{Picking up objects from a high shelf}

"Challenge-C" for the two robots was to accomplish "3. Placing and removing a sponge from a shelf." For this task, we decided to challenge the system by demanding that it remove a ceramic coffee cup-an object heavier and smoother than a sponge-from not any shelf but the highest shelf of a kitchen cabinet, and then place it within the confines of a small microwave oven. To do this, h+lamp bends (by tendon actuation) and extends (by linear actuation) to grip the coffee cup (Figure 7.A). The lamp fixture of the $h+$ lamp illuminates the interior space of the shelf (Figure 7.B), so that the user, if she or he wishes, can confirm that the robot is retrieving the desired object-an unnecessary behavior in a structured environment filled with machines and trained human operators, but one that may reassure a frail, untrained human navigating an unstructured home environment shared by a novel technology. Once the cup is retrieved, $h+$ lamp can maneuver the cup towards (Figure 7.C), and into (Figure 7.D) the microwave. The user then approaches the microwave by wheelchair, selects the cooking intensity and duration by buttons, and closes the door.

Indeed, in our design concept for these two robots and for the larger home+ ambition, we recognize the desirability of having the human "in the loop," acting as an active agent in the system rather than being served by it. This cooperation of users and machines is described, in philosophical thinking, by the "material-semiotics" of actor-network theory (ANT), by which people and things coexist as actors in a dynamic interplay of the animate and inanimate [10].

In addition to performing CS-PFP10 task "3," we also began exploring the capacity of h+lamp to assist users with "2. Donning/removing a jacket." In further studies, we will demonstrate how h+lamp transports a raincoat to a closet hook, and how h+lamp removes the coat from the closet hook and positions it conveniently on the back of the user. Again, in this scenario, there is a "humanin-the-loop": the robot is not doing all the work but facilitating the task for the user, who is cooperating with the machine (akin to the gentlemanly act of a man positioning a coat near a woman's upper-back to help facilitate her putting on the coat). Challenge- $\mathrm{C}$ 

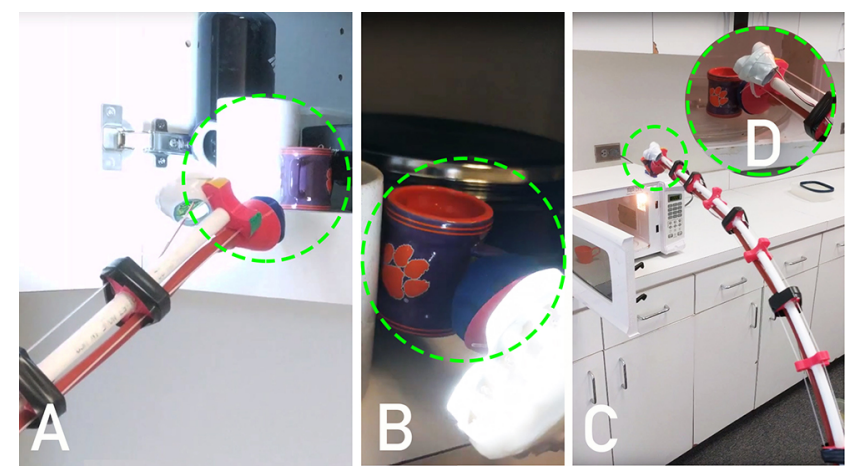

Figure 7: $\mathbf{h}+$ lamp collects a cup from the floor and delivers it to a microwave with the userâĂŹs cooperation.
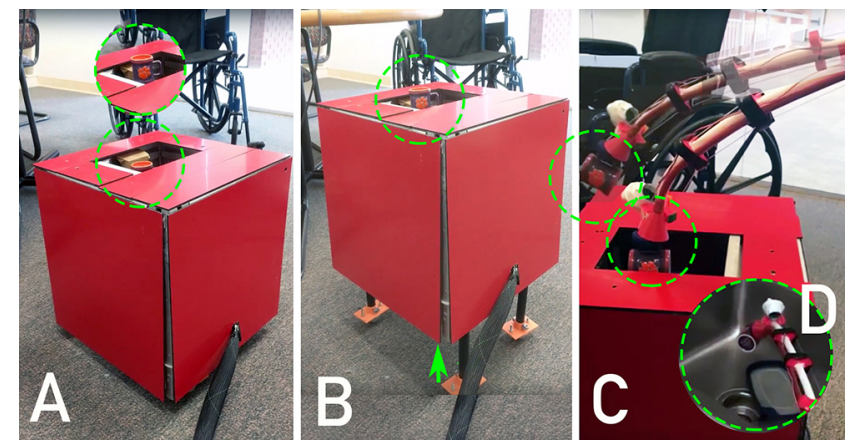

Figure 8: $h+$ cube collects a cup from the floor; then, $h+l a m p$ retrieves it from $h+c u b e$ and transfers it to the sink.

demonstrates how one of the two robots and the human cohabitant can work cooperatively to accomplish this task and many others like it within the home environment.

\subsection{Picking objects from floors and placing them in a sink}

"Challenge-D" for the two robots was a variation of accomplishing "8. Picking scarves off the floor" of Challenge-A. But unlike Challenge-A which involved collecting a sock from the floor and delivering it to a closet shelf-a task that $\mathrm{h}+$ cube can accomplish on its own-Challenge-D required both robots to work cooperatively in order to satisfy the demands of the task.

For Challenge-D, the ceramic coffee cup (used in the previous challenge) was first collected by $\mathrm{h}+$ cube as per the challenge involving picking up the pencil: the cup was collected by the gripper (Figure 8.A), elevated through the interior of the cube so that it surfaces at the top face of the cube (Figure 8.A-upper circle detail), and elevated again by way of the four linear actuators of the cube's legs (Figure 8.B). From this elevated position, the cup can reasonably be retrieved by the user in the wheelchair, as was the case in the pencil experiment. However, Challenge-D necessitates an additional behavior that is (literally) beyond the reach of $\mathrm{h}+$ cube: the cup (that had fallen to the floor) needs to be placed in the sink (for rinsing before use). Consequently, Challenge-D requires that $\mathrm{h}+$ lamp retrieve the cup from $\mathrm{h}+$ cube, and transport the cup to the sink (Figure 8.C-the sequence marked by the two circles). Finally, h+lamp sets the cup in the sink (Figure 8.D). Challenge-D demonstrates how the two robots can work cooperatively together to accomplish this task and many others like it within the home.

\subsection{Discussion of Lab Experiments}

In the course of developing the robot pair reported in this section, the research team observed adults ages 18-76 interacting with the prototypes using, for now, two control boxes, one box associated with each robot. (This control mechanism is a placeholder until the research team learns from future work the best control strategy for the robots, as stated previously.) Informed by our observations of this novel human-machine interaction, we intentionally bundled the eight CS-PFP10 tasks enumerated earlier in this section to create the four everyday domestic routines, or 'performances' elaborated in each of the preceding subsections. Arguably, we might have gained a certain clarity in our research design by focusing these experiment on each of the eight discrete CS-PFP10 tasks, not on four scenarios that umbrella them; however, at this stage of experimentation, the research team decided to sacrifice some clarity for gaining a broader understanding of the "human-robot ecology"-of how this novel cyber-human system might accomplish familiar, domestic routines in partnership with human users. We believe that bundling the discrete CS-PFP10 tasks into familiar domestic routines accomplished this objective. In future work, following extensive further study, we will return to measuring the performance of the user-home+ partnership in accomplishing the discrete CSPFP10 tasks, as compared to users accomplishing the same without home+. The research team created a video that predates this paper by some months but nevertheless is useful in capturing these four performances and overall functionality of the robot pair at home. (See https://vimeo.com/214498317.)

\section{FORMATIVE USABILITY EVALUATION}

A formative usability evaluation of the robot pair was conducted with older participants (Figure 9). Since engaging five users is expected to turn up $75 \%$ or more of the problems in the device being tested [27], five participants participated in this study. The five participants (three females, two males) ranged in age from 66-76 years of age, with a mean age of 70.8-years-old. All participants had cognitive and mobility limitations that were typical for their age group. The evaluation was conducted in our university lab. Approval for this evaluation was obtained from the appropriate institutional review board, and all five participants granted us permission to photograph and video record the evaluation activities. The study lasted 90 minutes.

At the onset of the study, the five participants entered our lab and sat around a meeting table. The team presented to the participants our video that was displayed on a large, wall-mounted computer display centered at the head of the table. The video introduced the home+ pair of robots, both in still photos and in video segments. Following the video viewing, the team offered a live demo of the hardware, and allowed the participants to interact with the robots using our control boxes. 
Table 3: Response by older adults to survey questions (higher sores suggest more usability or more user-satisfaction)

\begin{tabular}{|c|c|c|c|c|c|c|c|c|}
\hline & $\begin{array}{l}\text { I understand } \\
\text { how home+ } \\
\text { might help me. }\end{array}$ & $\begin{array}{l}\text { I understand how } \\
\text { home+ works on a } \\
\text { basic level. }\end{array}$ & $\begin{array}{l}\text { home+ will help } \\
\text { make routine tasks } \\
\text { easier to perform. }\end{array}$ & $\begin{array}{l}\text { I could envision } \\
\text { home+ in my } \\
\text { home. }\end{array}$ & $\begin{array}{l}\text { home+ will } \\
\text { make daily } \\
\text { chores fun. }\end{array}$ & $\begin{array}{l}\text { I like the look of } \\
\text { home+ - it's nice } \\
\text { looking. }\end{array}$ & $\begin{array}{l}\text { I think home+ } \\
\text { should operate } \\
\text { automatically. }\end{array}$ & $\begin{array}{l}\text { home+ could be } \\
\text { very helpful as I } \\
\text { grow older. }\end{array}$ \\
\hline Mean & 4 & 5 & 3.4 & 3.2 & 2.6 & 2.4 & 3.8 & 4.2 \\
\hline SD & 0.7 & 0 & 0.9 & 1.3 & 0.5 & 0.9 & 1.6 & 0.8 \\
\hline
\end{tabular}

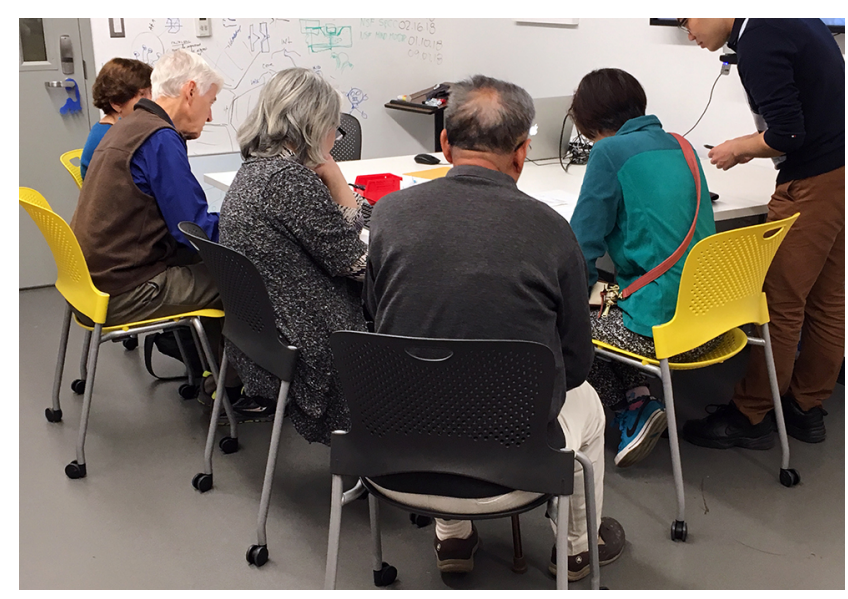

Figure 9: An instance from our usability evaluation with older adults.

Following the video and live demo, the participants were asked to complete a survey about home+ (the two robots) that included ten questions in total. The first eight questions (see Table 3) were in the form of a Likert-scale, for which each participant marked her/his preference on a scale of 1 (strongly disagree) to 5 (strongly agree). The first five questions asked participants about what they learned and felt about the robot pair after viewing the video, while the final three Likert-scale questions asked participants about the robot pair following the live demo. The final two questions of the survey were open questions that sought brief, written responses from the participants: Is there anything you would like to change in home+?, and Describe how you feel about home+.

Following the completion of the survey, team members convened a focus group that offered participants the opportunity to elaborate on their survey responses and offer further comments and suggestions. The survey results and focus group discussion were impactful on the iterative development of the prototypes, as elaborated below.

\subsection{Results}

Results of the Likert responses are presented in Table 3. All the questions are positively worded so that a higher score indicates either greater usability or more user-satisfaction. We recognize that phrasing the questions in the positive is known to lead to biased results [33]. We also recognize that, with a sample size of 5, scores are not statistically significant but only suggestive of usability and user satisfaction of the robots.
Participants ' high scores on how the robots function as a system (five scores of 5 for Q2) strongly suggest participants have a basic understanding of how the home+ robots work. On the two questions addressing "helpfulness" of the robots, participants' relatively high scores (mean of 4 for Q1 and mean of 4.2 for Q8) suggest that the home+ robots may prove helpful in their lives, both now and when they grow older. In short, we learned that participants understood how the robots function together with a user in performing routine domestic tasks, and that the robot pair has potential for helping these older adults now and as they grow yet older.

On questions of utility and user experience, participants' neutral scores (mean of 3.2 for both Q3 and Q4) suggest that the participants may or may not envision home+ in their homes, and that they perceive the robots as maybe making routine tasks easier to perform. However, low scores (mean of 2.6 for Q5 and 2.4 of Q6) suggest that home+ may not make daily chores especially fun or provide an aesthetically pleasing addition to their home's interior. Participants' moderately high score (mean of 3.8 for Q7) suggests with moderate confidence that participants would like home+ to operate (at least in part) autonomously. Indeed, as previously stated, the research team is developing and will compare three control scenarios: a completely-autonomous home+, a completely usercontrolled home+, and a mixed-control scenario of autonomy and user control. We intend, as a next-step in this research, to have healthcare professions inform the design of the home+ controls to suit the profiles of our target audience.

Following completion of the survey, we conducted a focus group during which participants expressed their excitement for the home+ robots. They praised the home+ concept, and firmly believed that elders will benefit from home+. Participants in the focus group suggested that they would be receptive to controlling the robots by futuristic interfaces such as eye movement or by brain wave function using EEG devices. The focus group also suggested that the robots might take too much physical space in the home and exhibiting a boxy form that was not sufficiently appealing to them (mean of 2.6 for Q6). Indeed, for these early home+ prototypes, the team strived to create: (a) robust functioning robots that could withstand some abuse, (b) robots that had ample interior room for all the hardware, (c) robots that were not likely to topple over and cause harm to users and each other, and (d) attractive robots, given the previous three parameters. We envision the footprint of the two robots becoming much smaller in area, and we also intend to study other form factors that might convey a more steam-lined, "domestic sensibility" to the home environment. Taken together, the survey results and focus group input suggest the promise of these two enabling technology prototypes. Our formative usability 
Table 4: Response by SMEs to survey questions (higher sores suggest more usability or more user-satisfaction)

\begin{tabular}{|c|c|c|c|c|c|c|c|c|c|}
\hline & $\begin{array}{l}\text { I understand } \\
\text { how home+ } \\
\text { might help } \\
\text { my patients. }\end{array}$ & $\begin{array}{l}\text { I understand } \\
\text { how home+ } \\
\text { works on a } \\
\text { basic level. }\end{array}$ & $\begin{array}{l}\text { home+ will help } \\
\text { make routine } \\
\text { tasks easier to } \\
\text { perform for my } \\
\text { patients. }\end{array}$ & $\begin{array}{l}\text { I can envision } \\
\text { home+ } \\
\text { in my } \\
\text { patients' } \\
\text { homes. }\end{array}$ & $\begin{array}{l}\text { home+ } \\
\text { could help } \\
\text { my patients } \\
\text { with their } \\
\text { daily chores. }\end{array}$ & $\begin{array}{l}\text { My patients } \\
\text { could become } \\
\text { productive } \\
\text { quickly using } \\
\text { home+. }\end{array}$ & $\begin{array}{l}\text { home+ has all } \\
\text { the functions } \\
\text { and capabilities } \\
\text { I expect it to } \\
\text { have. }\end{array}$ & $\begin{array}{l}\text { My patients } \\
\text { would like to } \\
\text { use something } \\
\text { like this. }\end{array}$ & $\begin{array}{l}\text { My patients would } \\
\text { need the support of } \\
\text { a technical person } \\
\text { to be able to use } \\
\text { home+. }\end{array}$ \\
\hline Mean & 4.3 & 4 & 3.9 & 3.5 & 4.3 & 3.4 & 3.4 & 3.8 & 4.1 \\
\hline SD & 0.8 & 0.9 & 0.8 & 0.8 & 0.8 & 0.8 & 0.7 & 0.9 & 1.0 \\
\hline
\end{tabular}

evaluation informed future research directions for advancing the functionalities and control of the home+ robots, their physical footprints, and their appearance.

\subsection{Limits of the user study}

As typical of user studies, our formative user study involved a small group $(n=5)$ so that the results, while suggestive of usability, are not statistically significant. For this early study, we also did not have access to participants who regularly use wheelchairs; consequently, we did not gain insights into the human-robot interactions with wheelchair users. Our study might also be criticized for a possible bias in posing all the survey questions in the positive; but eight Likert questions posed in the positive is arguably not so many to set a pattern that may lead participants to respond more positively. (Indeed, the participants during the focus group were quite vocal in their responses-at times critical, and at other times highly supportive of the concept and its promise.)

\section{A STUDY WITH CLINICIAL EXPERTS}

A few weeks after the study with older adults, our team conducted a study of the robot pair with twelve Subject Matter Experts (SMEs). The twelve SMEs ( 9 females and 3 males, ages 26-51 with a mean age of 37.6) are medical staff members of the Roger C. Peace Rehabilitation Hospital of the Greenville Health System. These SMEs represent the breadth of healthcare specialties that comprehensively reflects the needs and wants of the target population. Covering the gamut of healthcare providers for our target population, the participating SMEs were physical therapists, occupational therapists, speech therapists, physical medicine and rehabilitation physicians, and neuropsychologists. The evaluation was conducted at the hospital. Individually, each participant viewed the same video we presented to older adults in our previous user study. Following the video screening, each participant completed a survey. The questions posed are shown in Table 4. Following completion of the survey, each SME participated in an interview. Approval for this evaluation was obtained from the appropriate institutional review board.

\subsection{Results}

Results of the Likert responses are presented in Table 4. All the questions are positively worded so that a higher score indicates either more usability or more satisfaction. Overall, the SMEs "understood how home+ might help [their] patients" (mean of 4.3) and "how [home+] works on a basic level" (mean of 4.0). SMEs envisioned home+ helping their patients with daily chores (mean $=4.2$ ) but also imagined that their patients needed "the support of a technical person to be able to use home+" (mean = 4.1). The need for a technical person might be attributed to the significant physical and cognitive challenges faced by many of their patients. Overall, SMEs were somewhat less certain that patients would "like to use something like this" (3.8). Finally, SMEs collectively judged the home+ robot pair as not yet $($ mean $=3.4)$ equipped with all the functionality that they would expect them to have. This can be attributed to two very different issues: (a) the complexity of the physical and cognitive support their patients need, and (b) the lofty and ambitious desire for "surgical" precision in the robotic arm of h+lamp (e.g. for inserting a contact lens into one's eye). For the Likert-scale questions overall, SMEs scored the robots higher (more positively) than did the older adults, perhaps because the SMEs can see the potential of the concept.

In the interview activity, SMEs suggested joystick control of the robots for those in wheelchairs, and a smart phone app for other users. The SMEs also recommended voice control of the robots, and strongly suggested that a two-way voice or video monitor be integrated into the system. As the older adults offered, SMEs asked that the physical size of the cube and lamp be reduced, and that the packaging of them become less angular, more softened. SMEs liked the fact that the home+ pair could retrieve objects from a wide range of heights, floor to high cupboards. Our team's focus on routine kitchen scenarios was welcomed by the SMEs. A fuller discussion of these results will be presented in a future paper.

\section{CONCLUSION AND RECOMMENDATIONS}

This paper elaborated the iterative design development of $h+$ cube and h+lamp, and introduced how this cyber-human system performs as two robots-distinct, together, and with a human user in the loop-to accomplish routine tasks that constitute a proven index of independence.

The following recommendations are intended to generalize to other design teams developing assistive robots. These recommendations follow from discussions by our research team, informed by our prior work [15] and especially by the outcomes of the studies reported here: our team's observations of users of wide-ranging ages using the home+ robots to perform routine, domestic tasks as enumerated by CS-PFP10, our usability study with older adults, and our study with SMEs. Our recommendations: 
1. Assistive robotics do not require highly sophisticated, highcost technologies to accomplish routine, domestic tasks.

2. Given the likelihood that industry will bring many more domestic robots to market, designers developing such robots should think beyond single-function robots (e.g. those for vacuuming) to avoid over-populating everyday environments with single-function technologies.

3. An assistive robot system does not have to be housed in a single body; two or more robots with distinct behaviors can work well together as a distributed system with their human cohabitants.

4. Assistive robots do not have to look and behave like humans; they can exhibit their own behavior, which is artificial.

5. It is fruitful to develop prototypes with participants representing not only the targeted user groups but also their healthcare providers and perhaps, also, their more familial caregivers.

6. Designers should be mindful not to support but to enable and dignify users of assistive technologies.

Our next research activity invites SMEs and high-functioning older adults under their care to accomplish, with $\mathrm{h}+$ cube and $\mathrm{h}+\mathrm{lamp}$, a task (combining tasks 3.3 and 3.4 described earlier) involving: fetching a coffee mug, preparing coffee, drinking coffee, picking up a mug (after its accidental dropping) from the floor, and delivering the mug to the sink (for washing). We will also make the system mobile and interoperable, as accomplished in our prior work [9],[32] and as the capacity to both recognize furniture and other common objects (e.g. [4] and track human movement is increasingly understood (as in our efforts, [15]. While these technical advances are not trivial, what is less understood is how the familiar things of our dwellings can become robotic and enable us to age in place independently. This is the key contribution of the efforts reported here.

\section{ACKNOWLEDGMENTS}

This research is supported by the U.S. National Science Foundation under award IIS-1703267. The authors are grateful to Stephanie Tanner, Becky Snider, Lauren Mims and Breno Schwambach for their assistance with the usability study in the rehabilitation hospital.

\section{REFERENCES}

[1] 1995. Special Issue on Robots in Surgery. IEEE Engineering in Medicine and Biology Magazine 14 (1995).

[2] AARP. [n. d.]. Aging in Place: A Survey of Livability and Practices, http://assets.aarp.org/rgcenter/ppi/liv-com/ib190.pdf.

[3] Jenay M Beer, Cory-Ann Smarr, Tiffany L Chen, Akanksha Prakash, Tracy L Mitzner, Charles C Kemp, and Wendy A Rogers. 2012. The domesticated robot: design guidelines for assisting older adults to age in place. In Proceedings of the seventh annual ACM/IEEE international conference on Human-Robot Interaction. ACM, 335-342.

[4] Sean Bell and Kavita Bala. 2015. Learning visual similarity for product design with convolutional neural networks. ACM Transactions on Graphics (TOG) 34, 4 (2015), 98.

[5] J.J. Callahan. 1992. Aging in place. Generations 2, 16 (1992), 5-6.

[6] Neena L. Chappell and Zachary Zimmer. 1999. Receptivity to new technology among older adults. Disability and Rehabilitation 21 (1999), 222-230.
[7] Elaine Cress. [n. d.]. Rehab Measures: Continuous Scale Physical Functional Performance (CS-PFP), http://drelainecress.com/cs-pfp-test/.

[8] Sara J Czaja and Starr Roxanne Hiltz. 2005. Digital aids for an aging society. Commun. ACM 48, 10 (2005), 43-44.

[9] Carlos Henrique De Aguiar, Reza Fateminasab, Chase G Frazelle, Ryan Scott, Yixiao Wang, Michael B Wooten, Keith E Green, and Ian D Walker. 2016. The networked, robotic home+ furniture suite: A distributed, assistive technology facilitating aging in place. In Automation Science and Engineering (CASE), 2016 IEEE International Conference on. IEEE, 1067-1072.

[10] Brian Dellon and Yoky Matsuoka. 2007. Prosthetics, exoskeletons, and rehabilitation [grand challenges of robotics]. IEEE robotics \& automation magazine 14, 1 (2007), 30-34.

[11] Zhicong Deng, Martin Stommel, and Weiliang Xu. 2016. A Novel Soft Machine Table for Manipulation of Delicate Objects Inspired by Caterpillar Locomotion. IEEE-ASME Transactions on Mechatronics 21, 3 (2016), 1702-1710.

[12] David Fischinger, Peter Einramhof, Konstantinos Papoutsakis, Walter Wohlkinger, Peter Mayer, Paul Panek, Stefan Hofmann, Tobias Koertner, Astrid Weiss, Antonis Argyros, et al. 2016. Hobbit, a care robot supporting independent living at home: First prototype and lessons learned. Robotics and Autonomous Systems 75 (2016), 60-78.

[13] Terrence Fong, Illah Nourbakhsh, and Kerstin Dautenhahn. 2003. A survey of socially interactive robots. Robotics and autonomous systems 42, 3-4 (2003), 143-166.

[14] Birgit Graf, Matthias Hans, and Rolf D Schraft. 2004. Care-O-bot II-Development of a next generation robotic home assistant. Autonomous robots 16, 2 (2004), 193205.

[15] Keith Evan Green. 2016. Architectural robotics: ecosystems of bits, bytes, and biology. MIT Press.

[16] Erico Guizzo. 2014. So, where are my robot servants? IEEE Spectrum 51, 6 (2014), $74-79$

[17] Starr Roxanne Hiltz and Sara J Czaja. 2006. Introduction to the special issue on information systems for an aging society. ACM Transactions on Computer-Human Interaction (TOCHI) 13, 3 (2006), 309-312.

[18] Kevin Kelly. 1994. Out of control: The rise of neo-biological civilization. AddisonWesley Reading, MA.

[19] Ross A Knepper, Todd Layton, John Romanishin, and Daniela Rus. 2013. Ikeabot: An autonomous multi-robot coordinated furniture assembly system. In Robotics and Automation (ICRA), 2013 IEEE International Conference on. IEEE, 855-862.

[20] Larry Leifer. 1981. Rehabilitative robots. Robotics Age 3, 3 (1981), 4-15.

[21] Steven Levy. 1993. Artificial life: A report from the frontier where computers meet biology. Random House Inc.

[22] John Markoff. 2014. Brainy yes but far from handy. New York Times 1 (2014)

[23] Jessica Merino, Anthony L Threatt, Ian D Walker, and Keith Evan Green. 2012. Forward kinematic model for continuum robotic surfaces. In Intelligent Robots and Systems (IROS), 2012 IEEE/RSf International Conference on. IEEE, 3453-3460.

[24] W Mitchell. 1999. e-Topia: urban life, fim but not as you know it. MIT Press Cambridge.

[25] Elizabeth D Mynatt and Wendy A Rogers. 2001. Developing technology to support the functional independence of older adults. Ageing International 27, 1 (2001), 24-41.

[26] Alan Newell. 2003. Inclusive design or assistive technology. In Inclusive Design. Springer, 172-181.

[27] Jakob Nielsen. 1994. Usability engineering. Elsevier.

[28] Kwang-Hyun Park, Zeungnam Bien, Ju-Jang Lee, Byung Kook Kim, Jong-Tae Lim, Jin-Oh Kim, Heyoung Lee, Dimitar H Stefanov, Dae-Jin Kim, Jin-Woo Jung, et al. 2007. Robotic smart house to assist people with movement disabilities. Autonomous Robots 22, 2 (2007), 183-198.

[29] Joelle Pineau, Michael Montemerlo, Martha Pollack, Nicholas Roy, and Sebastian Thrun. 2003. Towards robotic assistants in nursing homes: Challenges and results. Robotics and autonomous systems 42, 3-4 (2003), 271-281.

[30] Ori Systems. [n. d.]. One Room, a Hundred Ways, https://orisystems.com/.

[31] Anthony L. Threatt, Jessica Merino, Keith Evan Green, Ian D. Walker, Johnell O. Brooks, Sean Ficht, Robert Kriener, Mary Mossey, Alper Mutlu, Darshana Salvi, George Schafer, Pallavi Srikanth, Peng Xu, Joe Manganelli, and Paul Yanik. 2012. A vision of the patient room as an architectural-robotic ecosystem. In 2012 IEEE/RS7 International Conference on Intelligent Robots and Systems. 3322-3323.

[32] Anthony L. Threatt, Jessica Merino, Keith Evan Green, Ian D. Walker, Johnell O. Brooks, and Stan Healy. 2014. An assistive robotic table for older and post-stroke adults: results from participatory design and evaluation activities with clinical staff. In Proceedings of the SIGCHI Conference on Human Factors in Computing Systems. 673-682.

[33] Shari Trewin, Diogo Marques, and Tiago João Vieira Guerreiro. 2015. Usage of Subjective Scales in Accessibility Research. In Proceedings of the 17th International ACM SIGACCESS Conference on Computers and Accessibility. 59-67.

[34] Sherry Turkle. 2011. Alone Together: Why We Expect More from Technology and Less from Each Other.

[35] Kazuyoshi Wada and Takanori Shibata. 2007. Living With Seal Robots-Its Sociopsychological and Physiological Influences on the Elderly at a Care House. 
IEEE Transactions on Robotics 23, 5 (2007), 972-980.

[36] I.D. Walker and K.E. Green. 2009. The Encyclopedia of Complexity and Systems Science. Springer, Chapter Continuum Robots, 1475-1485.

[37] Vincent Wall, Raphael Deimel, and Oliver Brock. 2015. Selective stiffening of soft actuators based on jamming. In 2015 IEEE International Conference on Robotics and Automation (ICRA). 252-257.

[38] Val Wang. [n. d.]. Two wheeled Robotic Table Balances Drinks, Segway-Style, https://www.popsci.com/scitech/article/2009-06/two-wheeledrobotic-table-balances-drinks-segway-style. 